\title{
R2D2 Model: Creating Diverse Online Instructions
}

\author{
Ke Zhang \\ Wayne State University
}

\begin{abstract}
With the increasing applications of online learning at all levels in training and education in Chinese-speaking countries and regions, together with the diversity of online learners in terms of age, background, and learning style, educators are faced with a critical challenge: how to design and implement online learning experiences to meet the varied needs of highly diverse online learners. This article introduces a highly practical, easy-to-apply model, the R2D2 model (Bonk \& Zhang, 2006, 2008) to the Chinese readers in particular, for the creation of diverse online learning activities. R2D2 (Read, Reflect, Display, and Do), is a new model particularly for online instructions. Focusing on online learners'different needs and preferences, this model suggests various learning activities in the above-mentioned four categories, to achieve knowledge acquisition, knowledge construction, knowledge display, knowledge application and knowledge transfer. The R2D2 model may also apply to the design and implementation of project based or problem based learning (PBL) (Bonk \& Zhang, 2008). This model is also one means to strategically select and integrate various emerging learning technologies to promote online learning for diverse learners.
\end{abstract}

Keywords: online learning, online instruction model, learning technology, diversity, instructional technology, R2D2, 


\title{
R2D2模型：创造多元化的网络教学
}

\author{
张珂 \\ 美国韦恩州立大学
}

【摘要】随着网络技术与资源在华语国家和地区教学与培训中的日益普及, 以及学习者 在年龄、背景、学习风格等各方面日益多元化, 教师和课程设计者们所面临的严峻挑战 之一即是如何针对多元化的生源、有效利用网络技术与资源来设计并教授网络课程。本 文旨在面向华语世界的网络教学设计者和教师, 介绍一个简明、实用、易于操作的网 络教学模型: R2D2模型 (Bonk \& Zhang, 2006, 2008), 用来指导设计不同类型的学习活 动, 为年龄、背景、学习风格、学习需求等多方差异的学习者提供多元化的学习机会。 R2D2模型是一种新兴的、特别针对网络教学的模型。该模型注重通过阅读 $(\mathrm{Read}) 、$ 深省 (Reflect)、展示(Display)、实践(Do)四类活动来实现知识获取 (knowledge acquisition)、知 识建构 (knowledge construction)、知识表征 (knowledge display), 知识应用 (knowledge application) 以及知识转移 (knowledge transfer)。该模型亦可用于设计和实施问题导向或项 目导向学习法 (PBL)(Bonk \& Zhang, 2008), 并且为广大网络教学的设计者们和教师们提 供了选择和应用各种不同教学技术的新思路和新方针。

【关键词】网络教学、教学多元化、网络教学模型、学习技术、教学技术、R2D2

\section{1. 前言}

根据中国互联网络信息中心 2008 年的 统计调查, 截至2007年12月, 中国网民数 已达到 2.1 亿人, 其中 3486 万人具有网络 学习经验, 网络教育也已经达到网民网络 使用总数的 $16.6 \%$ 。随着多种多样的网络 教学与培训在华语国家和地区日益普及, 以及学习者在年龄、背景、学习需求、学 习风格等各方面日益多元化, 教师和课程 设计者们所面临的严峻挑战之一即是如何 针对多元化生源与网络技术和资源来设计 并教授网络课程。新一代的学习者, 尤其 是那些 80 年代及以后出生的年轻一代对 信息技术、网络媒体和教学方式等各方面 有着全新的要求。网络教学所普遍面临的 一个重大问题将不仅是吸引新一代学习者 参加进来, 更要留住他们, 并有效降低他 们中途辍学的比例(Carr, 2000; Diaz, 2002;
Frankola, 2001)。因此, 我们有必要在充 分认识传统的学习风格等相关理论与科研 文献的基础上 (Felder \& Brent, 2005; Kolb, 1984; Lawrence, 1993), 进一步根据学习者的 特点提供一个新的理论框架, 针对性地指 导、开发、设计多样化的网络学习。

网络及其相关技术的迅猛发展使得网络 教学日益呈现出开放、互动、多元、共享 和共建等特征。比如Web2.0 和开源网络软 件的出现, 为广大教育工作者提供了日益 增多的、甚至是免费的网络资源和渠道。 他们可以通过博客(blog)、维基(wiki)等来 发表和分享知识、技术、专长、学术观点 等, 并可充分利用这些全新的教学技术创 建个性化的网络学习资料和课件 (Bonk \& Zhang, 2008, 2006)。面对如此丰富的网 络技术和资源, 如何对其进行选择、灵活 多样地应用于网络教学、创造多元化的网 
络学习环境, 来满足各类学员的不同需求? 这无疑是教师、学者和课程设计人员所正 在面临的一个关键问题。

本文旨在面向网络教学的设计者和教 师, 介绍一个简明、实用、易于操作的网 络教学模型: R2D2模型 (Bonk \& Zhang, 2006，2008), 用来指导设计不同类型的学 习活动, 为年龄、背景、学习风格、学习 需求等多方差异的学习者提供多元化的学 习机会。该模型旨在帮助网络教学人员选 择适当的技术, 并将其巧妙融入不同的教 学活动中, 从而针对性地提供有效的网络 教学。随着网络教学的不断普及, R2D2模 型亦可视为一个简明、有效、可行的理论 框架以指导广大教学人员适应网络这一新 的教学方式所带来的各种挑战和变化。

\section{2. 学习风格和偏好}

各种学习风格 (learning style) 和偏好 (learning preference) 的理论与模型由来已 久, 且影响深远 (Santo, 2006; 康淑敏, 2003；谭顶良，1994，1996，1998)。尽管 目前暂无有力研究表明学习者的学习风格 与其网络学习成败密切相关(Santo, 2006), 学习风格和偏好理论一直以来为因材施教 的教学实践提供了坚实的理论基础。本文 仅简短评论与 R2D2模型密切相关的有关理 论或模型。Kolb（1984）将有效的学习周 期分为四个不同阶段: 切身体验、具体经 历, 观察思考, 抽象概念和主动实践、验 证。在此基础之上, McCarthy（1987）进 一步构建了 4MAT系统, 并确定了四类不同 的学习风格: 创新型、分析型、常识型和 动力型。根据McCarthy的研究, 创新型的 学习者注重知识和认知的个人意义, 经常 通过自身参与和与他人的讨论来寻求知识 的意义。而分析型学习者则关注于对事实 的理解、分析和评判, 善于抽象思维。常 识性的学习者对事物是如何运作的格外好 奇, 而动力性学习者则常常在自发的探索 中学习、发现和进步。

Fleming 和 Mills (1992a, 1992b) 也 定义了四种类型的学习风格, 分别为
视觉型(Visual)、听觉型(Auditory)、读 写型 (Reading、writing) 和运动实践型 (Kinesthetic)。因而又称VARK模型。具体 而言, 视觉型学习者喜欢图形、表格、 流程图等视觉辅助。但是值得一提的是, 现极为普及的教育技术中的视频、电影、 短片、网上直播和 PowerPoint 等并不在 Fleming 和 Mills 所定义的视觉辅助范 畴, 因为它们通常不仅有视像也包括声 频、动作等多元因素。而听觉学习者在学 习过程中通常受益于讲座和口头讲解。读 写型学习者则经常在阅读或编写文本、资 料信息时学到更多知识。而运动实践型的 学习者通常在各种动手型的教学活动中, 如角色扮演、辩论、实验和模拟、仿真 等, 积极受益。

简言之, 各类教学活动与学习情境都 有其独特的优点。Bonk、Kim 和 Zeng (2006) 近期的研究表明, 网络教学的需求 正经历着重大变革, 并不断引入诸如主动 学习 (active learning)、基于项目或问题 的学习 (PBL亦称问题导向或项目导向学 习) 、实境学习 (authentic learning)、虚拟 团队、网络协作学习等多样的、以学习者 为中心的教学方式。随着日益增多的 80 年 后、90年后的新一代学习者加入网络学习 的行列, 网络生源亦在年龄、社会经历、 背景和对网络科技的熟知程度上有了更 多、更大的差异。这些在西方被称为 $Y$ 世 代 (Generation Y)、千禧世代 (Millennial) 或网络世代 (net generation) 的年轻学习者 酷爱网络、视听、多媒体等高新科技电子 产品（如iPod、iPhone、掌上电脑PDA等） , 熟悉Web2.0 技术与网站（如博客blog、 在线共享内容亦称聚合内容RSS、维基、社 会性网络或社交网络软件Social Network） , 并热衷于所谓e时代的社交方式 (Dede, 2005; Oblinger，2003）。因此他们对网 络学习也就有着类似的要求和期待: 教学 方式应该高度互动, 活动与内容应丰富多 彩并个性化, 而非单纯的文字阅读、被动 地听讲座和读讲义。然而我们目前的网络 教学距离他们的需求与期待仍有相当的距 离。尽管各类技术、资源无疑为课程设计 
者和教师提供了大量可能的、新颖的教学 方式; 但与此同时, 太多的选择亦令人无 所适从, 不知从何着手。而目前普及常用 的学习管理系统 (LMS) 和课程管理系统

(CMS) 并未能充分利用广大网络资源与技 术, 导致多数网络课程仍严重依赖于单一 的文本阅读(Bonk \& Zhang, 2008)。为解决 这一难题, Bonk和笔者(Bonk \& Zhang, 2006 ，2008)共同创建了R2D2这一模型，以便广 大课程设计者和教师针对多元化的网络生 源, 设计和实施丰富多彩的、行之有效的 教学活动。本文旨在结合中国教育现状, 为广大中文读者, 尤其是积极从事网络教 学设计、发展与实施的教育工作者们, 介 绍这一模型, 提供一些新思路、新点子以 创建丰富多彩的网络课程。

\section{R2D2模型}

如图1所示, R2D2 模型是专门为网络教学 所创建的高度实用型模型。不同于同名的 (Jost, Mumma, \& Willis, 1999)或是有类似名 称的R2D5模型 (Pederson, 2005), 此模型并 非教学设计模型, 也因此并不着重于教学 设计的具体过程或步骤。R2D2模型注重于 引导设计者和教师针对各异的学习者, 正 视他们在学习风格、偏好、能力、需求与 年龄等各方面的差异, 有效选择不同类型 的学习任务、技术资源或者教学活动, 以 实现高效的、多元化的网络学习。与4MAT 和VARK模型相似, 该模型确认了四类学习 活动: 阅读(Reading)、深省(Reflecting)、 展示(Displaying)及实践(Doing)。不同的 是, 该模型是专为网络教学或混合式学习

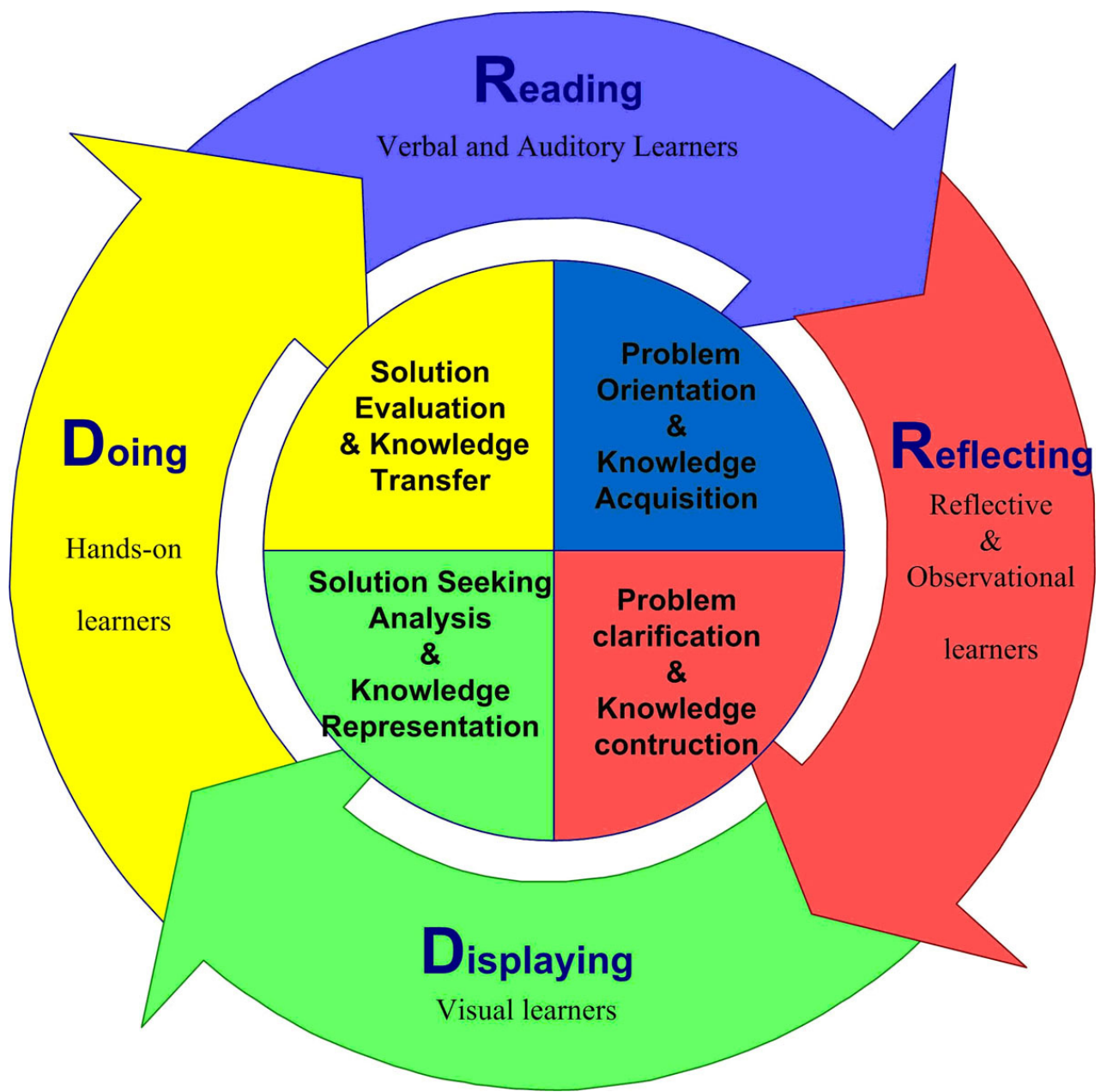

图 1: R2D2模型：各阶段、类型的教学活动与PBL（问题解决）的步骤 Bonk \& Zhang（2008），第6页 
(blended learning)所创建; 而且R2D2模型重 视促进学习者深省, 并将写作和其它编写 类的活动归于深省类，而非阅读类。

听觉型学习者偏好于字词、声音及口头 或书面解释。观察思考型学习者常通过观 察、反思、复习、自我测试及总结类写作 等活动从不同视角考察、判断和学习。而 视觉型学习者偏好于图片、图表、图形、 概念图 (concept map)、流程图、照片、影 像、视频和各种实物及步骤展示。实践型 学员无疑偏向于动手操作、角色扮演、游 戏、模拟仿真等动感类型的教学活动。如 表格1所示, R2D2模型融合不同网络信息技 术, 尤其是免费的网络资源, 针对各类学 习者提出了一系列形式各异学习活动。

该模型不但可用于指导课程设计以满 足不同学习者的特点与需求, 亦可用于问 题导向或项目导向学习法 (PBL) (Bonk \& Zhang, 2008)。如图1和表格2 (在下页) 所 示, 该模型所列举的四类活动亦可视为解
决问题 (problem solving)的四个动态步骤 (Bonk \& Zhang, 2008, 第6-8页)。

举例而言, 学习者可以在阅读与深 省过程中熟悉了解问题或项目 (problem orientation) 并获取知识 (knowledge acquisition), 然后在深省思维和视觉展 示类的活动中进一步澄清问题 problem clarification)、建构知识 (knowledge construction), 在视觉展示与动手实践的过 程中分析、探寻问题的解决方法(solution seeking) 并实现知识表征 (knowledge representation), 最后进行解决方法的评 估 (solution evaluation) 并实现知识转移 (knowledge transfer)。

\section{R2D2模型的应用}

R2D2这一动态的、非线性的模型包括四 类或四阶段教学活动: 阅读、深省、展示 及实践。根据该模型, 教师或课程设计者 可以针对不同学习者或者不同学习阶段的

表格1: R2D2模型中的学员类型、教学活动与相应技术范例 教学活动类型与学员类型 网络教学活动及技术范例

阅读: 听觉型 播客(podcast)、网络PDF文档、语音文档、门户网站(portal) 、Powerpoint、课程公告、常见问题解答（FAQ）、时事通 讯、新闻简报、电子书、网络杂志、网络图书馆、网络资源 库等

深省：观察思考型

博客、实时聊天(synchronous chat)、网上测验、作文帮助工 具、电子档案(e-portfolio)、异步讨论(asynchronous discussion) 、线上复习、线上自我测试、反思性写作、专家录像和表演 等

展示: 视觉型

各类图形、表格、图像、概念图、时间表及其制作工具、互 动新闻 (interactive news)、视频、虚拟实地考察、动画、图 片、白板(whiteboard)、视频会议、视频博客等

实践: 动手实践型

仿真、模拟、游戏、实验、维基、数码化故事创作(digital storytelling)、影像制作、时事案例、情境学习、协作学习、角 色扮演、网络辩论、虚拟社区等 
表格2: R2D2模型应用于PBL或问题解决

\begin{tabular}{lll}
$\begin{array}{l}\text { PBL过程 或 } \\
\text { 问题解决的步骤 }\end{array}$ & 主要任务 & 可能的R2D2阶 \\
\hline 问题陈述或定义 & 段或类型 \\
& 清楚了解问题、课题及具体任务, 确认 & 阅读 \\
& 解决问题所需信息, 寻找相关数据、资 & 展示 \\
寻求解答 & 料, 寻求专家意见等 & \\
& 集思广益, 评估可能的资源并将任务与 & 阅读 \\
& 资源进行先排序 & 深省 \\
寻找资料、资源、信息等 & 读写视听等途径收集、整理资料与信息 & 实读 \\
以解决问题 & 并分析 & 深省 \\
& & 展示 \\
评估解决方案 & 评估、反思、比较问题的解决方案 & 实践 \\
& & 深省 \\
& & 展示 \\
\hline
\end{tabular}

需求来选择、采用不同类型的教学活动。 有必要特别指出的是, 该模型所列举的各 项活动, 几乎无一例外、或多或少都是针 对不止一种学习风格和偏好。正如人们通 常会有不止一种的学习风格或偏好, 又如 人们在学习不同学科或内容时可能偏好不 同的学习方法, 该模型也无意将各种各样 的学习活动进行简单划一的切割性分类。 本文将这些活动进行分类的目的是强调它 们分别主要针对哪一类学习者或学习风 格、偏好, 以方便教师和设计者们有针对 性地选择、整合各式各样的教学技术与活 动。

作为一个高度动态的网络教学模 型, R2D2模型所提倡的四类教学活动: 阅 读、深省、展示及实践之间的关系是非线 性的, 并且互相关联的。尽管各类学习活 动中可能在难度等方面的差异, 它们相互 之间并不一定存在高级、低级或类似的层 级关系。换言之, 实践类活动不一定必须 安排在深省或展示类活动之后。举例而 言, 网络教学活动并不一定要从阅读开 始, 而实践类教学活动也不一定非得在最
后阶段才能有效进行。根据学习者背景和 教学需求, 教师和课程设计师可以从丰富 多彩的R2D2活动中选择合适的教学活动应 用于教学的不同阶段; 而这些教学活动可 以分别是阅读、深省、展示、实践类, 也 可以综合两种或多种类型的教学活动同时 进行。

\section{1. 阅读类网络教学活动}

阅读类教学活动着重于知识获取并主 要服务于听觉型学员, 所以并不限于传统 的、纯文本类阅读。在这一阶段的教学活 动中, 学习者探寻事实、掌握基本概念, 获取知识, 为以下各阶段如深省反思、展 示、知识应用、转移与构建奠定基础。这 一类型或阶段的学习可以通过阅读、汶览 和聆听相关的资料资源、线上讲座、网上 讨论与客座专家聊天等方式实现。通过网 上教程、录音、音频文件、小组讨论等多 样化的活动, 学习者可以从多种渠道, 包 括视觉、语音等获取信息与知识。

播客, 作为数字广播技术的一种正在 中国教育界日益普及（罗永昌, 王基一, 
2006; 赵英芳, 黎加厚，2008）。学员可 以自动订阅 (RSS feed) 和录制并在网络传 播各种声讯或影像资料来获取信息、资料 并实现知识共享。虚拟课堂和网络会议也 是获取知识的有效途径; 若结合网络辅导 (web tutoring)、文件共享、问卷调查、即时 民意测验等功能, 更可实现教师与学习者 以及学习者之间的多层次的互动。

网络寻宝(scavenger hunt)和网络主题探 究(Webquest) 也是常见的阅读类活动。教师 可以让学习者阅读、聆听、观看或汶览制 定的学习资料, 也可以让学习者搜寻与某 主题相关的资料然后阅读、讨论、总结。 各种阅读类活动可以在教师指导下进行; 教师也可以让学习者根据个人兴趣在指定 范围内有选择性地阅读, 甚至制定更为开 放型的学习任务一此类活动当然对有一定 网络学习经验的和主动自觉的学习者可能 更容易接受, 而初学者可能需要一定的适 应过程和更多的教师指引。常见问题解答 和课程公告栏也是有效的阅读类教学活 动。

实时聊天, 尤其是带语音功能的聊天 室也是网络教学中常用的、有效的网络学 习和交流方式。教师们也可以考虑用聊天 室功能与学习者进行一对一的实时测试和 疑难解答。外语和其它社会科学类的课程 还可以考虑采用网络新闻简报、杂志、博 客、播客等方式让学生了解更多现实社会 的时事、动态、文化、现象等, 并引导他 们进行更深入的思考。在网络教学中实现 知识的获取的方法有很多种, 而教师的适 当指引尤为重要。

\section{2. 深省类网络教学活动}

R2D2模型中的深省类活动侧重于激发学 习者的深刻思维和深层反思, 写作这一重 要的思维表达方式也因此被归为此类。网 络教学提供了很多传统课堂所无法实现的 学习机会。比如说, 在异时讨论过程中, 参与者可以有充分的时间思考、草拟并修 改他们的讨论文章和回复。这些非即时 的、延迟反应(delayed response)对于深层探
讨、和批判性思维有极大的促进作用, 尤 其是针对有争议的单一专题或相关多专题 的深入探讨。教师们也可以引导学生创建 相关的网络资源库 (online resource library) ，汇集他们平时发现和收集的相关资料, 以进一步激励自觉、自发的学习和知识共 建。

在阅读的基础上, 网络协同写作也正 成为日益普及的网络教学活动 (魏慧娟, 2007; 王伟，2005）。越来越多的网络用 户, 包括不少学习者和教育工作者们已经 拥有了他们自己的博客。作为网络课程中 的一项学习任务, 博客也可以有效促进学 习者对所学内容的深层思考。而小组博 客、班级博客, 甚至跨班级的、跨国合作 的博客更是可以让学习者们在相互交流的 过程中发现新观点、共同创建新知识。 在网络课程中, 设立批判性朋友 (critical friends)也深受学习者欢迎。在笔者的多门 网络课程中, 批判性朋友屡次被学生们评 为做受欢迎和最有效的教学活动之一。教 师可以按学习者的兴趣、背景指定 2-4人 互为批判性朋友, 这一安排可以贯穿整个 课程, 也可以定期更换让学习者接触到更 多同学。批判性朋友的主要任务是相互批 判性审阅对方的网络发言、作业初稿、博 客文章等, 分享心得体会, 相互鼓励, 并 以专业态度提出建设性意见和建议, 以期 共同提高。深省类活动也包括让学习者以 短文、博客、播客、PowerPoint等方式进 行阶段性或某专题的总结, 并由教师、同 学、客座专家等提供书面或口头评语、意 见。在课程结束前要求学习者总结他们自 身的学习过程和成果, 包括批判性朋友在 内的各式学习活动和作业等也能进一步促 进学习者的深省, 并帮助教师和课程设计 师根据学习者的反馈适当修改网络课程。

网络课程也可以通过 Breeze $\mathrm{BM}$ 、 Elluminate ${ }^{\mathrm{TM}}$ 、Wimba ${ }^{\mathrm{TM}}$ 等多媒体实时交流 系统在虚拟课堂内对指定的播客、网络研 讨会等进行深省、讨论。教师可以现场进 行物理、化学等实验, 并实时加入相关讲 义、问题讨论或学员笔记等。这些资料均 
可存档并重复使用。在此类活动中学员们 有更强的参与感。在某些学科, 如物理、 化学、体育、心理咨询、教师培训等, 线 上示范和与学员的即时互动尤为重要也极 其有效。类似的, 教师们也可以充分利用 网络论坛、网络会议等技术进行虚拟法庭 (mock trial)、辩论和角色扮演等活动来促 进学员的辩证思维和深层思考, 并提供更 多协同学习的机会以实现知识共建和分 享。学习者还可以充分利用网络技术与资 源, 采访与专业或课题相关的专家、实业 家, 并结合自身感想撰写关于该专业或课 题的研究报告。他们也可以根据某一具体 概念、原则、课题、讲义、讲座、专家报 告、案例等, 联系实际问题设计可行性方 案, 或对既定项目、方案提出修改意见。 教师们可以充分利用视频、录像等资源引 导学员对现实生活中的情境、案例进行反 思、深省。借助流媒体 (stream media), 学习者可以参加网络实时研讨会、手术直 播、戏剧表演等多种线上直播活动; 若条 件允许, 学习者还可以在第二人生(Second $\mathrm{Life}^{\mathrm{TM}}$ )等三维立体的网络环境下进行虚拟 的实习和更为系统的情境学习。随着更多 实时互动学习技术的普及, 网络课程可以 提供更多虚拟实习的机会。

在学习者自我测试、自我评估、自我 反省等活动中教师们还可以通过 “你知道 吗” 这类简单的问题提示来引导学员们在 学习过程中暂停并深入思考。此类活动在 学习者不能及时获得大量教师反馈时显得 尤为重要。与之类似, 提供答案范例、以 前或现在学习者的优秀作业供参考、示范 也是有效促进学习者深省的方法。如果创 建光荣榜或明星榜, 在每次作业后及时甄 选优秀学员作业、作品及教师评语供本班 学习者参考, 还能进一步调动学习者的积 极性和主动性。

\section{3. 展示类网络教学活动}

R2D2模型的第三部分强调用各种方式让学 习者充分展示所学成果, 并提供形式各异 的视觉辅助以着重满足视觉类学习者的偏
好与需求。网络教学中此类活动包括提供 和引导学习者创作、分享并应用图片、图 表、表格、视频、动画等视觉辅助工具, 以及在科学教育中使用数学、科学符号和 动态模拟工具等。此类活动与深省类活动 会有明显重叠，因为它们常有类似过程： 先宏观演示概念或原理, 然后让学习者对 其实际应用进行深入探讨和思考。在网络 教学中, 有很多工具、资源和方法可用于 视觉展示类的教学活动。在实时交流时, 教师可以在互动型白板上手写文字并辅之 以色彩、箭头、注解、图片等方式突出重 点、解析疑难。教师也可以让学习者汶览 相关的网上视频来学习新概念、步骤和过 程。在商、法、医、药、教育等学科教学 活动中, 案例视频或录像等被常用于展示 现实案例和重点讨论其中的关键概念和重 要问题。教师们更可以充分利用网络资源 让学习者进行虚拟的实地考察, 并且接下 来可以进一步进行定点抛针式讨论与教学 (anchored instructions)。

视频博客和动画也是常见的视觉展示类 学习活动。探险性的博客可以提供最新和 互动式的故事、游记和科学实验或是探险 考察的视频。因此, 学习者宛如身临其境, 可以在动态的实时情境中学习, 而不仅仅 是从书本或网络上进行简单的文本阅读。 而动画常用于更清楚地阐述、解释一个概 念。暂停、快进、重播等功能让学员们可 以根据自身需要, 有选择性的观看某些片 断和反复播放, 并且有更多时间思考。视 频、虚拟之旅 (virtual tour)、探险性的博客 和动画都可为学习者提供一个更加真实的 学习情境 (Collins, 1990), 而且让他们可以 在现实或近乎真实的情境中学习(Herrington \& Oliver, 1997; Reeves, Herrington, \& Oliver, 2002; Herrington, Oliver, \& Reeves, 2003)。

借助于各种各样的网络视觉展示工具和 渠道, 视觉类的学习活动可引导学习者将 在阅读类活动（R2D2模型中的第一部分） 中掌握的新知识、新概念, 经过深刻反省 与思考 (R2D2模型中的第二部分) 抽象 化、视觉化, 并通过有创意的视觉展示进 
一步深化学习。有关研究表明, 让学习者 编创抽象化的视觉展示, 以表达他们各自 对知识的理解, 并解构新旧知识间的联系 能让他们对所学知识印象更深刻(Cognition and Technology Group at Vanderbilt, 1990 , 1991)。例如, 学生们可以创建一个概念 图, 以此来总结一篇论文、一个专题等。 学生们还可以互相交流、比较各自编绘的 概念图、流程图和虚拟之旅, 并探讨其差 异和共性, 以及他们对同一内容的不同理 解和诠释。通过这些视觉展示类的教学活 动学生们将逐步建立更强的思辨能力和自 我评估的能力。作为一个高度视觉化的工 具, 网络为教、学双方提供了前所未有的 新方式和新机会。随着越来越多的新兴科 技的出现 (如Web2.0技术等), 教育工作 者必须积极探索如何充分利用这些技术与 资源, 并引导学习者在网络学习过程中逐 步成长为积极、主动、自觉的学习者。

\section{4. 实践类网络教学活动}

实践类教学活动可能是目前常见的网络 教学中最为缺乏却又最为急需的。 R $2 \mathrm{D} 2$ 模 型的这一部分 (或阶段) 侧重于创造各种 实践机会, 让学习者实际应用所学的知识 和技能, 并在应用中学习、进步、提高。 此类活动不仅能满足动手实践型学习者的 需求, 更是促进各种各样的学习者理论联 系实际、学以致用, 实现知识转移和问题 解决等高层次学习目标所必需的环节。

案例和仿真是实践型活动的代表。置身于 现实案例或仿真情境中, 学习者不但可 以应用所学知识解决问题, 还可以比照 专家提案和现实案例中所采纳的解决方 案, 并从对比和反思中学习、提高。举例 而言, 利用网络门诊案例库(http://www. mymedcases.com/product/MedCasesFrame. asp), 医学院的学习者可以从网络获取病 人的相关资料, 要求病人进行有关测试, 并根据测试结果开方确诊, 然后可以参照 比对专家意见和解释说明。学习者还可以 参加多方虚拟会诊, 观摩或参与虚拟手 术。这些仿真练习无疑将帮助他们在面对
真正的病人之前做好更充分的准备。基于 案例的学习活动可以在案例真实度、复杂 度和难度等方面给学员们带来不同程度的 挑战。教师们可以采用全真、实时案例 (Theroux, Carpenter, \& Kilbane, 2004 ）, 由专职案例作家跟踪报道某公司动向, 让学习者 (如商学院、新闻学院等) 及时 分析该案例的发展动态、提出方案解决问 题。这类活动的编创无疑对时间、费用和 案例编写经验都有相当高的要求，但是可 以重复使用以降低成本。另一种方式是通 过教师指定某课题或调研方向, 由学员们 自己设计研究方案、设定数据收集工具（ 如调查所用问卷、民意测验题、访问稿 等）来收集资料、分析数据并撰写案例报 告。笔者在台湾高校的网络课程中进行过 此类教学活动, 让学习者全面参与调研的 全过程, 充分调动其学习的积极性和自主 性, 并激发他们的创造力、思辨能力、团 队合作精神和应用知识解决问题的能力 (Zhang, Hung, \& Peng, 2005)。学习者也可以 采访名人或普通人以了解某一特殊社会现 象; 应用相关科技编创多媒体、互动式作 品 (如wiki 项目、视频播客、e-portfolio 等), 并邀请专家评审 (Oliver, Herrington, \& Reeves, 2006; Oliver \& McLoughlin, 1999; Oliver, Omari, \& Herrington, 1998）。教师 们可以充分利用网络资源, 让学员们采用 现实世界的真实数据进行市场调研、科学 实验、决策分析等活动。笔者和合作者 $(\mathrm{Ou}$ \& Zhang, 2006)曾介绍了多种教学方法以 利用网络上真实的、实施、动态数据库来 进行多学科的、跨学科的教学与科研。在 这类学习活动过程中, 学习者和教师之间 可以运用多种网络技术 (如网络视频、网 络电话、聊天室、论坛等) 进行实时交流 和讨论。

随着Web2.0 的日益普及，第二人生等由 用户共同创建的虚拟世界与社区, 因其体 验性和娱乐性正吸引越来越多的教育界学 者、专家的关注。在中国, 三维立体虚拟 平台HiPiHi也在游戏里开辟了青少年的专 区, 传播诸如宇宙飞船如何发射之类的科 普知识等; 而U21高教在线也在第二人生 
里建立了虚拟校园。这些虚拟世界给教育 界提供了新的平台来创建丰富多彩的、生 动有趣的学习情境、任务和各种阅读、深 省、展示和实践到机会; 而我们需要更多 的实践和研究来探求如何最大程度上实现 其教、学潜力。

\section{5. 再议R2D2模型}

阅读、深省、展示和实践这四类教学 活动是R2D2模型的切入点。每一类活动着 重针对该类型的学习者或学习偏好。如果 在班级中某一类学习者占绝大多数, 或是 某一类学习活动最合适某一课题, 那么教 师可以注重选择某一类学习活动, 并为学 习者提供各种不同的此类学习任务。但是 更多情况下, 面对多元化的网络生源, 课 程设计师和授课人员应有针对性地选择多 种类的教学活动以满足各类学员的不同需 求。虽然R2D2并不是传统意义上的教学设 计 (instructional design)模型, 但是网络课程 的设计者和教师可以依据该模型为背景、 风格、偏好各异的学习者设计不同类型的 学习活动。R2D2并非线性模型, 所以学习 者并不一定要从阅读到深省再到展示最后 才是实践。在某些教学阶段, 不同类型的 教学活动也可以同时进行。笔者期望R2D2 模型在网络课程设计中的应用将把远程教 育的技术整合带入新的高度, 为网络教学 带来大量积极、正面的变革, 改变人们对 于网络教学简单、枯燥的偏见, 提高学习 者对网络学习的满意度和增进网络学习效 果。

\section{参考文献}

罗永昌，王基一（2006）。发挥Podcast 在提高学生信息素养中的作用。中国远 程教育, 04 .

康淑敏 （2003）。学习风格理论一一西方 研究综述。山东外语教学; 2003年03期 谭顶良（1994）。论学习风格及其研究 价值 $[\mathrm{J}]$; 南京师大学报 (社会科学版); 1994年03期； 46-50。

谭顶良（1996）。学习风格理论应用于教
育心理学教学的尝试。心理科学; 1996 年02期； 125-126。

谭顶良（1998）。学习风格的研究及其在 教学实践中的应用。江苏高教; 1998年 05期; 56-58。

王伟（2005）。B1og在基于网络的研究性 学习中的作用。中国远程教育, 2005, (03).

魏慧娟（2007）。博客促进现代远程教育 培训双向交互的研究。首都师范大学, 2007.

赵英芳, 黎加厚 (2008)。 Podcast在英语 学习中的应用。软件导刊 (教育技术), 2008, (04).

中国互联网络信息中心 (2008). 中国互联 网络发展状况统计报告。2008年3月5 日在互联网获取：http://www.cnnic.cn/ uploadfiles/doc/2008/1/17/104126.doc.

Bonk, C. J., Kim, K. J., \& Zeng, T. (2006). Future directions of blended learning in higher education and workplace learning settings. In C. J. Bonk \& C. R. Graham (Eds.). Handbook of blended learning: Global perspectives, local designs. San Francisco, CA: Pfeiffer Publishing.

Bonk, C. J., \& Zhang, K. (2006). Introducing the R2D2 model: Online learning for the diverse learners of this world. Distance Education, 27(2), 249-264.

Bonk, C. J., \& Zhang, K. (2008). Empowering online learning: 100+ Activities for online reading, reflecting, displaying, and doing. San Francisco, CA: Jossey-Bass.

Carr, S. (2000, February 11). As distance education comes of age, the challenge is keeping the students. The Chronicle of Higher Education, A39-A49. Retrieved June 6, 2005, from http://chronicle.com/ $\mathrm{prm} /$ weekly/v46/i23/23a00101.htm

The Cognition and Technology Group at Vanderbilt (1990). Anchored instruction and its relationship to situated cognition. Educational Researcher, 19(6), 2-10.

The Cognition and Technology Group at Vanderbilt (1991). Technology and the 
design of generative learning environments. Educational Technology, 31(5), 34-40.

Collins, A. (1990). Cognitive apprenticeship and instructional technology. In L. Idol \& B. F. Jones (Eds.), Educational values and cognitive instruction: Implications for reform. Hillsdale, NJ: Lawrence Erlbaum Associates.

Dede, C. (2005). Planning for neomillennial learning styles: Implications for investments in technology and faculty. Retrieved June 29, 2005, from http://www.educause.edu/ content.asp?page_id $=6069 \&$ bhcp $=1$

Diaz, D. P. (2002, May/June). Online drop rates revisited. The Technology Source Archive. Retrieved September 2, 2008, from http:// technologysource.org/article/online_drop_ rates_revisited/

Felder, R.M., \& Brent, R. (2005). Understanding student differences. Journal of Engineer Education, 94(1), (pp. 57-72). Retrieved June 28, 2005, from http://www.ncsu. edu/felder-public/Papers/Understanding Differences.pdf

Fleming, N. D., \& Mills, C. (1992a). Not another inventory, rather a catalyst for reflection. To improve the academy, (11), 137-149.

Fleming, N. D. \& Mills, C. (1992b).VARK a guide to learning styles. Retrieved October 11, 2005, from http://www.vark-learn.com/ English/index.asp

Frankola, K. (2001). Why online learners drop out. Workforce, 80, 53-58.

Herrington, J., \& Oliver, R. (1997). Multimedia, magic and the way students respond to a situated learning environment. Australian Journal of Educational Technology, 13(2), 127-143.

Herrington, J., Oliver, R., \& Reeves, T. (2003). Patterns of engagement in authentic online learning environments. Australian Journal of Educational Technology, 19(1), 59-71.

Jost, M., Mumma, P., \& Willis, J. (1999). R2D2: A constructivist/interpretivist instructional design model. In J. Price et al. (Eds.),
Proceedings of Society for Information Technology and Teacher Education International Conference 1999 (pp. 14891494). Chesapeake, VA: AACE.

Kolb, D. A. (1984). Experiential learning: Experience as the source of learning and development. Englewood Cliffs, New Jersey: Prentice-Hall.

Lave, J., \& Wenger, E. (1991). Situated learning: Legitimate peripheral participation. New York: Cambridge University Press.

Lawrence, G. (1993). People types and tiger stripes: A practical guide to learning styles, (3rd ed.). Gainesville, FL: Center for Applications of Psychological Types.

McCarthy, B. (1987). The 4MAT system: Teaching to learning styles with right/left mode techniques (Revised ed.). Barrington, IL: EXCEL.

Oblinger, D. (2003). Understanding the "new students". Educause (July/August 2003). 37-47.

Oliver, R., Herrington, J., \& Reeves, T. C. (2006). Creating authentic learning environments through blended learning approaches. In C. J. Bonk \& C. R. Graham (Eds.). Handbook of blended learning: Global Perspectives, local designs, (pp. 502-515). San Francisco, CA: Pfeiffer Publishing.

Oliver, R., \& McLoughlin, C. (1999). Curriculum and learning-resources issues arising from the use of Web-based course support systems. International Journal of Educational Telecommunications, 5(4), 419-436.

Oliver, R., Omari, A., \& Herrington, J. (1998). Exploring student interactions in collaborative World Wide Web computerbased learning environments. Journal of Educational Multimedia and Hypermedia, 7(2/3), 263-287.

Ou, C., \& Zhang, K. (2006). Teaching with Databases: Begin with the Internet. TechTrends, 50(5), 46-51.

Pedersen, D. (2005). Online development made 
easy-At least easier. Distance Learning, 2(3), 22-23.

Reeves, T. C., Herrington, J., \& Oliver, R. (2002). Authentic activities and online learning. In A. Goody, J. Herrington \& M. Northcote (Eds.), Quality conversations: Research and development in higher education, Volume 25, 562-567.

Santo, S. (2006). Relationships between learning styles and online learning: Myth or reality? Performance Improvement Quarterly, 19(3), 73-88.

Theroux, J., Carpenter, C., \& Kilbane, C. (2004). Experimental online case study for a breakthrough in student engagement: Focus group results. Journal of Asynchronous Learning Networks, 8(3). Retrieved October 10, 2005, from http://www.sloan-c.org/ publications/jaln/v8n3/v8n3 theroux.asp

Zhang, K. (2005). China's online education: Rhetoric and realities. In A. A. CarrChellman (Ed.), Global perspectives on E-learning: Rhetoric and reality. 21-34. Thousand Oaks, CA: Sage Publications.
Zhang, K., \& Hung, J. (2006). E-learning in Taiwan: Policies, practices, and problems. International Journal of Information and Communication Technology Education, 2(1), 37-52.

Zhang, K., Hung, J., \& Peng, S. (2005, October). Moderating online collaborations during various tasks in a project-base learning environment, paper presented at the Annual Meeting of the Association for Educational Communications and Technology, Orlando, FL.

\section{联系作者}

张珂, 博士

美国韦恩州立大学

电邮: ke.zhang@wayne.edu

Ke Zhang, Ph.D.

Wayne State University

Email: ke.zhang@wayne.edu 